\title{
On the regularity of null-controls of the linear 1-d heat equation
}

\author{
Sorin $\mathrm{Micu}^{\text {a }}$ \\ ${ }^{a}$ Facultatea de Matematica si Informatica, Universitatea din Craiova, Al. I. Cuza 13, Craiova 200585, Romania. \\ Enrique Zuazua b,c \\ bIkerbasque, Basque Foundation for Science, Alameda Urquijo 36-5, Plaza Bizkaia, 48011, Bilbao, Basque Country, Spain \\ ${ }^{\mathrm{c}}$ BCAM - Basque Center for Applied Mathematics, Bizkaia Technology Park 500, 48160, Derio, Basque Country, Spain
}

\begin{abstract}
The $1-d$ heat equation in a bounded interval is null-controllable from the boundary. More precisely, for each initial data $y^{0} \in L^{2}(0,1)$ it corresponds a unique boundary control of minimal $L^{2}(0, T)$-norm which drives the state of the $1-\mathrm{d}$ linear heat equation to zero in time $T>0$. This control is given as the normal derivative of a solution of the homogeneous adjoint equation whose initial data $\widehat{\varphi}^{0}$ minimizes a suitable quadratic cost functional. In this Note we analyze the relation between the regularity of the initial datum to be controlled $y^{0}$ and that of $\widehat{\varphi}^{0}$. We show that, if $y^{0}$ has only one Fourier mode, the corresponding $\widehat{\varphi}^{0}$ does not belong to any Sobolev space of negative exponent. This explains the severe ill-posedness of the problem and the lack of efficiency of most of the existing numerical algorithms for the numerical approximation of the controls, and in partricular the slow convergence rate of Tychonoff regularization procedures.
\end{abstract}

Sur la regularité des contrôle HUM pour l'équation de la chaleur linéaire.

\section{Résumé}

L'équation de la chaleur $1-d$ dans un intervalle borné est contrôlable depuis le bord. Plus précisément, pour tout $y^{0} \in L^{2}(0,1)$ il existe un unique contrôle frontière de norme $L^{2}(0, T)$ minimale qui conduit la solution de l'équation linéaire de la chaleur à zéro à l'instant $T>0$. Ce contrôle est donné par la dérivée normale au bord d'une solution de l'équation adjointe avec une donnée initiale $\widehat{\varphi}^{0}$ qui minimise une fonction coût quadratique. Dans cet article nous étudions la relation entre la régularité de $y^{0}$ et celle de $\widehat{\varphi}^{0}$. Nous montrons que, si $y^{0}$ a une seule fréquence de Fourier, la fonction $\widehat{\varphi}^{0}$ correspondante n'appartient à aucun espace de Sobolev d'exposant négatif. Ce fait explique le caractère mal posé du problème et l'inefficacité de la plupart des algorithmes numériques existants et en particulier la convergence lente des méthodes de régularisation de type Tychonoff.

Email addresses: sd_micu@yahoo.com (Sorin Micu), zuazua@bcamath.org (Enrique Zuazua). 


\section{Version française abrégée}

L'équation de la chaleur $1-d$ dans un intervalle borné (1) est contrôlable depuis le bord. Plus précisément, pour tout $y^{0} \in L^{2}(0,1)$, il existe un unique contrôle frontière $v$ de norme $L^{2}(0, T)$ minimale qui conduit la solution $y$ de l'équation linéaire de la chaleur à zéro à l'instant $T>0: y(T) \equiv 0$.

Ce contrôle est donné par la dérivée normale au bord d'une solution de l'équation adjointe (2) avec une donnée initiale $\widehat{\varphi}^{0}$ qui minimise la fonction coût quadratique $\mathcal{J}$ donnée par (3). Le minimum de cette fonction est atteint sur un espace de Hilbert ad-hoc $\mathcal{H}$ defini à partir de l'inégalité d'observabilité (4) du système adjoint.

Cet espace est difficile à caractériser en termes classiques. Il est connu qu'il est plus large que tout espace de Sobolev d'indice négatif à cause du fort phenomène régularisant de l'équation de la chaleur. Ce fait est lié au caractère mal posé du problème au sens que, en particulier, les méthodes numériques usuelles ont souvent des vitesses de convergence très lentes ([1,6]). C'est, par exemple, le cas des méthodes d'approximation basées sur la régularisation de Tychonoff.

Dans cette Note nous donnons une réponse précise à cette question en démontrant que les minima de la fonctionnelle $\mathcal{J}$ sont souvent aussi irreguliers que l'espace $\mathcal{H}$ le permet. Nous faisons cela à travers une analyse soigneuse de la relation entre la régularité de $y^{0}$ et celle de $\widehat{\varphi}^{0}$. Nous montrons, en particulier, que, si $y^{0}$ a une seule fréquence de Fourier, la fonction $\widehat{\varphi}^{0}$ correspondante n'appartient à aucun espace de Sobolev d'exposant négatif car ses coefficients de Fourier croissent de manière exponentielle à hautes fréquences. Notre preuve est basée sur une analyse des minimiseurs à l'aide des estimations fines sur la suite de fonctions biorthogonales des exponentiels intervenant dans le développement en série de Fourier des solutions.

Ceci donne une explication définitive au caractère mal posé du problème et à l'inefficacité de la plupart des algorithmes numériques existants et en particulier la lente convergence des méthodes de régularisation de type Tychonoff qui, pour établir une vitesse de convergence, ont besoin d'un minimum de regularité Sobolev des minima.

\section{Introduction}

Given $T>0, y^{0} \in L^{2}(0,1)$ and $v \in L^{2}(0, T)$, we consider the non-homogeneous 1-d heat equation

$$
\begin{cases}y_{t}(t, x)-y_{x x}(t, x)=0 & x \in(0,1), t \in(0, T) \\ y(t, 0)=0, \quad y(t, 1)=v(t) & t \in(0, T) \\ y(0, x)=y^{0}(x) & x \in(0,1) .\end{cases}
$$

System (1) is well known to be null-controllable (or controllable to zero) in time $T$ for all $T>0$. More precisely, for each $y^{0} \in L^{2}(0,1)$ there exists a control $v \in L^{2}(0, T)$ such that the corresponding solution of (1) verifies $y(T, \cdot)=0$. The control of minimal $L^{2}(0, T)$-norm is unique and can be characterized through the adjoint system as described below.

Given $\varphi^{0} \in L^{2}(0,1)$ we consider the adjoint heat equation

$$
\begin{cases}\varphi_{t}(t, x)+\varphi_{x x}(t, x)=0 & x \in(0,1), t \in(0, T) \\ \varphi(t, 0)=\varphi(t, 1)=0 & t \in(0, T) \\ \varphi(T, x)=\varphi^{0}(x) & x \in(0,1) .\end{cases}
$$


In view of the regularizing properties of the heat equation, the map $\varphi^{0} \rightarrow\left(\int_{0}^{T}\left(\varphi_{x}\right)^{2}(t, 1) d t\right)^{\frac{1}{2}}$ is a norm in $L^{2}(0,1)$. Let $\mathcal{H}$ be the completion of $L^{2}(0,1)$ with respect to this norm. Now, we introduce the functional $\mathcal{J}: \mathcal{H} \rightarrow \mathbb{R}$ given by

$$
\mathcal{J}\left(\varphi^{0}\right)=\frac{1}{2} \int_{0}^{T}\left|\varphi_{x}\right|^{2}(t, 1) d t-\int_{0}^{1} y^{0}(x) \varphi(0, x) d x,
$$

$\varphi$ being the solution of $(2)$ with initial data $\varphi^{0}$.

For all $y^{0} \in L^{2}(0,1), \mathcal{J}$ is coercive in $\mathcal{H}$. This is a deep result that requires of the following observability inequality: For all $T>0$ there exists $C>0$ such that

$$
\|\varphi(0)\|_{L^{2}(0,1)}^{2} \leq C \int_{0}^{T}\left|\varphi_{x}(1, t)\right|^{2} d t, \quad \forall \varphi^{0} \in L^{2}(0,1) .
$$

This kind of result is by now well known also in the multi-dimensional case. We refer to [7] for an updated account on the developments in this context.

The functional $\mathcal{J}$ being coercive in $\mathcal{H}$, in addition to being continuous and strictly convex, it has a unique minimizer $\widehat{\varphi}^{0} \in \mathcal{H}$. The normal derivative of the solution of the adjoint system associated to this minimizer yields the control of minimal $L^{2}(0, T)$-norm. Summarizing, the following holds (see, for instance, [4]).

Proposition 1 Let $T>0$ be given. For each $y^{0} \in L^{2}(0,1)$ there exists a unique control $v \in L^{2}(0, T)$ of minimal $L^{2}(0, T)$-norm for equation (1) and $v=\widehat{\varphi}_{x}(\cdot, 1)$, where $\widehat{\varphi}$ is the solution of the adjoint problem (2) with initial data $\widehat{\varphi}^{0} \in \mathcal{H}$, the minimizer of (3). Moreover, the map $\mathcal{G}: L^{2}(0,1) \rightarrow \mathcal{H}, \mathcal{G}\left(y^{0}\right)=\widehat{\varphi}^{0}$, is linear and continuous.

Despite of this clear functional description and characterization of this optimal null control, as reported in $[1,6]$, the problem of computing $\widehat{\varphi}^{0} \in \mathcal{H}$ by minimizing (3) is severely ill-posed. This comes from the fact that the space $\mathcal{H}$ is very large. In fact, due to the regularizing effect of the heat equation, one can see that any distribution in a negative order Sobolev space $H^{-s}(0,1)$, with support away from $x=1$, belongs to $\mathcal{H}$, whatever $s>0$ is. Therefore, for a given $y^{0} \in L^{2}(0,1)$, the minimizer of $\mathcal{J}$ may have very low regularity and it may be difficult to capture it numerically with accuracy and robustness.

The main aim of this article is to investigate the regularity of $\mathcal{G}\left(y^{0}\right)=\widehat{\varphi}^{0} \in \mathcal{H}$ and to show that, indeed, for regular initial data $y^{0}$, as it is for instance the case when $y^{0}$ is constituted by one single Fourier mode, the corresponding minimizer $\mathcal{G}\left(y^{0}\right)$ of $\mathcal{J}$ does not belong to any Sobolev space of negative exponent.

This clearly expresses the severe ill-posedness of the problem and also shows why classical methods, based on Tychonoff regularization (see [3]), fail to be efficient. Indeed, the existing results about the convergence rates for Tychonoff regularization require a minimal regularity of the minimizer $\mathcal{G}\left(y^{0}\right)=\widehat{\varphi}^{0}$ of $\mathcal{J}$ in some Sobolev space, something that, as described above, is not fulfilled.

\section{Analysis of the operator $\mathcal{G}$}

Let us introduce some notation. The eigenvalues of the 1-d Dirichlet Laplace operator are $\lambda_{n}=n^{2} \pi^{2}$ and the corresponding eigenfunctions $\Phi^{n}=\sin (n \pi x)$, for every $n \in \mathbb{N}^{*} . \Lambda=\left(e^{-\lambda_{n} t}\right)_{n \geq 1}$ denotes the family of the corresponding real exponential functions and, for any $T>0, E(\Lambda, T)$ is the closure of the 
algebraic span of $\Lambda$ in $L^{2}(0, T)$. It is known that, for any $T>0$, there exists a unique biorthogonal family $\left(\theta_{T}^{m}\right)_{m \geq 1}$ to $\Lambda$ in $L^{2}(0, T)$ such that $\left(\theta_{T}^{m}\right)_{m \geq 1} \subset E(\Lambda, T)$. Moreover, this biorthogonal sequence has minimal $L^{2}(0, T)$-norm.

Let $v$ be the control of minimal $L^{2}(0, T)$-norm corresponding to $y^{0}$ and $\widehat{\varphi}^{0}=\mathcal{G}\left(y^{0}\right)$ given by Proposition 1. As we have said before, our aim is to study the regularity of $\widehat{\varphi}^{0}$ and we do this by expanding it in Fourier series

$$
\mathcal{G}\left(y^{0}\right)=\widehat{\varphi}^{0}(x)=\sum_{m \geq 1} b_{m} \sin (\pi m x)
$$

and analyzing the behavior of the Fourier coefficients $b_{m}$ that can be characterized as follows.

Theorem 2.1 Let $y^{0}=\sum_{n \geq 1} a_{n} \sin (\pi n x) \in L^{2}(0,1)$. The Fourier coefficients $b_{m}$ from (5) are given by

$$
b_{m}=\frac{(-1)^{m}}{m \pi} \sum_{n=1}^{\infty}(-1)^{n} \frac{a_{n}}{2 n \pi} e^{-n^{2} \pi^{2} T} d_{n}^{m} \quad \forall m \geq 1
$$

where $d_{n}^{m}=\left\langle\theta_{T}^{n}, \theta_{T}^{m}\right\rangle_{L^{2}(0, T)}$ for all $m, n \geq 1$.

Proof: From the minimal norm property of $\theta_{T}^{n}$ in $L^{2}(0, T)$, we deduce that the control of minimal $L^{2}(0, T)$ norm corresponding to $y^{0}$ is given by the formula

$$
v(t)=\sum_{n=1}^{\infty}(-1)^{n} \frac{a_{n}}{2 n \pi} e^{-n^{2} \pi^{2} T} \theta_{T}^{n}(T-t) .
$$

If $\widehat{\varphi}$ is the solution of (1) with initial data $\widehat{\varphi}^{0}$ given by $(5), v=\widehat{\varphi}_{x}(\cdot, 1)$ and $(6)$ follows immediately.

In order to evaluate the Fourier coefficients $b_{m}$ of $\mathcal{G}\left(y^{0}\right)$ we need to estimate the quantities $d_{k}^{n}$. This is done in the following key result whose proof requires of significant technical developments that can be found in [4].

Theorem 2.2 There exist a positive integer $n_{0}$ and a positive constant $C>0$, independent of $k$ and $m$, but depending of $T$, such that

$$
\begin{aligned}
& \left|d_{k}^{m}\right| \leq C e^{\pi(k+m)} \quad \forall k, m \geq 1 \\
& \left|d_{k}^{m}\right| \geq \frac{k m}{32\left(k^{2}+m^{2}\right)} e^{\pi(k+m)} \quad \forall m, k \geq n_{0} .
\end{aligned}
$$

Sketch of the proof: We use a strategy similar to the one used in [2], where the norms $\left\|\theta_{T}^{n}\right\|_{L^{2}(0, T)}=\sqrt{d_{n}^{n}}$ are evaluated. It consists in "truncating" the infinite matrix $G=\left(d_{k}^{m}\right)_{k, m \geq 1}$ by considering the minimal norm biorthogonal $\left(\theta_{\infty}^{m}\right)_{1 \leq m \leq N}$ to the finite family $\Lambda_{N}=\left(e^{-\lambda_{n} t}\right)_{1 \leq n \leq N}$ in $L^{2}(0, \infty)$. In this case we have an explicit formula for the quantities $d_{k}^{m}(N, \infty)=\left\langle\theta_{\infty}^{m}, \theta_{\infty}^{k}\right\rangle_{L^{2}(0, \infty)}$. Then, a perturbation argument allows to estimate $d_{k}^{m}(N, T)=\left\langle\theta_{T}^{m}, \theta_{T}^{k}\right\rangle_{L^{2}(0, T)}$, where $\left(\theta_{T}^{m}\right)_{1 \leq m \leq N}$ is the minimal norm biorthogonal to $\Lambda_{N}$ in $L^{2}(0, T)$. Finally, by letting $N$ to tend to infinity, we obtain the desired estimates for $d_{k}^{m}$. 


\section{Control theoretical consequences}

In this section we deduce some consequences of Theorems 2.1 and 2.2 for the controllability of (1). Let us consider the case in which the initial data $y^{0}$ of (1) to be controlled is the $n$-th eigenfunction $\Phi^{n}$ of the Laplace operator. The following result is a direct consequence of (6) and estimates (8)-(9).

Corollary 3.1 Let $n \geq n_{0}$ and $\left(b_{m}^{0, n}\right)_{m \geq 1}$ be the Fourier coefficients of $\mathcal{G}\left(\Phi^{n}\right)$ from (5). Then

$$
\left|b_{m}^{0, n}\right| \geq \frac{1}{64 \pi^{2}\left(n^{2}+m^{2}\right)} e^{-\pi^{2} T n^{2}+\pi(m+n)} \quad \forall m \geq n_{0} .
$$

The constants $C$ and $n_{0}$ are those given by Theorem 2.2, are independent of $n$ and $m$ but depend of $T$.

Remark 1 Estimate (10) shows that, for any $n \geq n_{0}$, the initial data $\mathcal{G}\left(\Phi^{n}\right)$ of (2), which gives the control of minimal $L^{2}(0, T)$-norm for the eigenfunction $\Phi^{n}$, has a very low regularity. Indeed, for any $m \geq 2 \pi T n^{2}$, we obtain that $\left|b_{m}^{0, n}\right| \geq e^{\frac{\pi}{2} m}$ and therefore $\mathcal{G}\left(\Phi^{n}\right)$ does not belong to any Sobolev space of negative order. $\square$

The following result concerns the regularity of the control of minimal $L^{2}(0, T)$-norm for arbitrary initial data in $L^{2}(0,1)$.

Corollary 3.2 For any $y^{0} \in L^{2}(0,1)$ and $\varepsilon>0$ there exists $y_{\varepsilon}^{0} \in L^{2}(0,1)$ and a positive constant $C$, depending only of $T$ and $\varepsilon$, such that $\left\|y^{0}-y_{\varepsilon}^{0}\right\|_{L^{2}(0,1)}<\varepsilon$ and

$$
\left|b_{\varepsilon, m_{k}}^{0}\right| \geq \frac{C}{m_{k}^{2}} e^{\pi m_{k}} \quad \forall k \geq 1,
$$

$\left(b_{\varepsilon, m}^{0}\right)_{m \geq 1}$ being the Fourier coefficients of $\mathcal{G}\left(y_{\varepsilon}^{0}\right)$ and $\left(m_{k}\right)_{k \geq 1}$ an increasing sequence of positive integers.

Proof: Let $\mathcal{G}\left(y^{0}\right)=\sum_{m=1}^{\infty} b_{m}^{0} \sin (m \pi x)$ and $n_{0}$ be the positive integer from Theorem 2.2. We define $y^{1}=y^{0}+\varepsilon \sin \left(n_{0} \pi x\right)$ and remark that $\mathcal{G}\left(y^{1}\right)=\mathcal{G}\left(y^{0}\right)+\varepsilon \mathcal{G}\left(\Phi^{n_{0}}\right)$, where $\mathcal{G}\left(\Phi^{n_{0}}\right)$ was studied in Corollary 3.1. If $\left(b_{m}^{1}\right)_{m \geq 1}$ are the Fourier coefficients of $\mathcal{G}\left(y^{1}\right)$, we deduce that

$$
b_{m}^{1}=b_{m}^{0}+\varepsilon b_{m}^{0, n_{0}}=b_{m}^{0}+\varepsilon \frac{(-1)^{n_{0}+m}}{2 n_{0} m \pi^{2}} e^{-n_{0}^{2} \pi^{2} T} d_{m}^{n_{0}} \quad m \geq 1 .
$$

Consequently, $\max \left\{\left|b_{m}^{0}\right|,\left|b_{m}^{1}\right|\right\} \geq \frac{\varepsilon\left|d_{m}^{n_{0}}\right|}{4 n_{0} m \pi^{2}} e^{-n_{0}^{2} \pi^{2} T}$ and, by taking into account (9) from Theorem 2.2, we deduce that at least one of the sequences $\left(b_{m}^{0}\right)_{m \geq 1}$ or $\left(b_{m}^{1}\right)_{m \geq 1}$ has a subsequence which verifies (11). If this is $\left(b_{m}^{0}\right)_{m \geq 1}$, we choose $y_{\varepsilon}^{0}=y^{0}$. Otherwise, we take $y_{\varepsilon}^{0}=y^{1}$ and the proof ends.

Remark 2 Corollary 3.2 shows that the set of initial data $y^{0}$ whose controls of minimal $L^{2}(0, T)$-norm are given by minimizers $\mathcal{G}\left(y^{0}\right)$ of $\mathcal{J}$ which do not belong to any Sobolev space of negative order is dense in $L^{2}(0,1)$.

\section{Acknowledgements}

The first author was partially supported by Grant MTM2008-03541 funded by MICINN (Spain) and Grant 420/2008 of CNCSIS (Romania). Part of this work was done during the first author's visit to Basque Center for Applied Mathematics and he would like to thank the members of the center for their 
kindness and warm hospitality. The second author has been partially supported by Grant MTM200803541 of the MICINN, Spain, the ERC Advanced Grant FP7-246775 NUMERIWAVES, ESF Research Networking Programme OPTPDE and the Grant PI2010-04 of the Basque Government.

\section{References}

[1] C. Carthel, R. Glowinski and J.-L. Lions, On exact and approximate Boundary Controllabilities for the heat equation: A numerical approach, J. of Optimization Theory and Applications, 82 (1994), 429-484.

[2] H. O. Fattorini and D. L. Russell, Exact controllability theorems for linear parabolic equation in one space dimension, Arch. Rat. Mech. Anal., 43 (1971), 272-292.

[3] J.-L. Lions, Optimal Control of Systems Governed by Partial Differential Equations, Springer- Verlag, Berlin, 1971.

[4] S. Micu and E. Zuazua, An introduction to the controllability of partial differential equations, Chapter in Quelques questions de théorie du contrôle, Ed. Tewfik Sari, Collection Travaux en Cours, Editions Hermann 2005, 69-157.

[5] S. Micu and E. Zuazua, Regularity issues for the null-controllability of the linear 1-d heat equation, preprint, 2010.

[6] A. Münch and E. Zuazua, Numerical approximation of trajectory controls for the heat equation through transmutation, J. Inverse Problems, 26(8) 085018 (2010) (39 pp.), doi:10.1088/0266-5611/26/8/085018.

[7] A E. Zuazua, Controllability and Observability of Partial Differential Equations: Some results and open problems, in Handbook of Differential Equations: Evolutionary Equations, vol. 3, C. M. Dafermos and E. Feireisl eds., Elsevier Science, 2006, 527-621. 